\title{
The Characteristics and Outcomes of Abernethy Syndrome in Korean Children: A Single Center Study
}

\author{
Eun Sil Kim, Ki Wuk Lee*, and Yon Ho Choe \\ Department of Pediatrics, Samsung Medical Center, Sungkyunkwan University School of Medicine, Seoul, \\ *Department of Pediatrics, Seoul National University Bundang Hospital, Seoul National University School of Medicine, \\ Seongnam, Korea
}

Purpose: Abernethy malformation is a rare condition, which was first described in 1793 as a congenital extrahepatic porto-systemic shunt (CEPS) directing splanchnic blood flow into the inferior vena cava. Eighty cases have been published so far that reported CEPS, while in Korea, very few cases have been reported. Through this study, we present 6 cases of patients diagnosed with CEPS at Samsung Medical Center and compare these with other such cases published in France and China.

Methods: We reviewed clinical, laboratory, and imaging data of 6 children with CEPS in our pediatric clinic between 2004 and 2017.

Results: A total of 6 children with CEPS was included in this study, namely, one with type 1a, two with type $1 \mathrm{~b}$, and three with type 2 CEPS. The most common presenting symptom was gastrointestinal bleeding (50.0\%). Therapeutic interventions included shunting vessel ligation (16.7\%) in type 2 CEPS and liver transplantation (16.7\%) in type 2 CEPS patient with suddenly developed hepatic encephalopathy.

Conclusion: There is no consensus guideline for the optimal management of patients with CEPS. Large-sample studies regarding CEPS are needed to evaluate the characteristics of patients with CEPS and determine the treatment guideline for CEPS.

Key Words: Congenital extrahepatic portosystemic shunt, Korean children

\section{INTRODUCTION}

Abernethy malformation is a rare condition, which was first described in 1793 as a congenital extrahepatic porto-systemic shunt (CEPS) directing splanchnic blood flow directly into the inferior vena cava [1]. Clinical manifestations, especially in children, are extremely variable, involving hepatic, neurological, pulmonary, metabolic, and other systems. Morgan and Superina classified CEPS into two types

Received : April 15, 2018, Revised : June 12, 2018, Accepted : June 22, 2018

Corresponding author: Yon Ho Choe, Department of Pediatrics, Samsung Medical Center, Sungkyunkwan University School of Medicine, 81 Irwon-ro, Gangnam-gu, Seoul 06351, Korea. Tel: +82-2-3410-3527, Fax: +82-2-3410-0043, E-mail: i101016@skku.edu

Copyright (c) 2019 by The Korean Society of Pediatric Gastroenterology, Hepatology and Nutrition

This is an open-access article distributed under the terms of the Creative Commons Attribution Non-Commercial License (http://creativecommons.org/licenses/by-nc/4.0/) which permits unrestricted non-commercial use, distribution, and reproduction in any medium, provided the original work is properly cited. 
[2] (Table 1). In a type 1 shunt, portal blood is diverted completely into the inferior vena cava, with absence of the portal vein. Type 1 Abernethy malformation can be further divided into subtype la and lb. In subtype la, the superior mesenteric vein and the splenic vein do not connect and thus there is no anatomic portal vein, whereas in subtype $1 b$, the superior mesenteric vein and splenic vein connect to form a portal vein, which then drains into a systemic vein [3]. In type 2 Abernethy malformation, the portal vein is intact, but a side-to-side anastomosis with the inferior vena cava leads to shunting. Therefore, structurally, type 2 shunt is not characterized by true absence of the portal vein. A type 2 shunt is more common in males and is rarely associated with other malformations [4].

In type 1 CEPS, which is characterized by absence of intrahepatic portal vein, the portal blood is completely drained into the systemic venous circulation. In type 2 CEPS, the portal blood is partially drained into the systemic venous circulation, and a hypoplastic intrahepatic portal vein is present [5].

Eighty cases have been published so far that reported extrahepatic portosystemic shunts [6]; while in Korea, very few cases have been reported as CEPS. Through this study, we present 6 cases of the patients diagnosed with CEPS at Samsung Medical Center while comparing these with the other cases published in France and China.

\section{MATERIALS AND METHODS}

From June 2004 to April 2017, 6 children were investigated for CEPS, treated, and followed-up at the Samsung Medical Center. The clinical sources of all CEPS cases were reviewed, including electronic medical records, histopathologic material (if available), and imaging and laboratory findings. The presence of shunt was confirmed by abdominal ultrasonography (US), computed tomography (CT), magnetic resonance imaging, or a combination of these techniques. Investigations for complications of the shunt and associated anomalies included liver function tests; estimation of the plasma ammonia levels and serum alpha-fetoprotein levels; coagulation studies; echocardiography; imaging studies of liver tumors and liver histology. The portosystemic shunts were classified anatomically based on the results of the imaging studies. The follow-up investigations consisted of clinical, biochemical, and US examinations.

\section{RESULTS}

The main findings are summarized in Table 2, including the clinical presentation, type of shunt, associated anomaly, associated abnormalities, treatment and outcome. In the review, 6 patients were identified with a mean age of 1.5 years at the initial diagnosis (range, 11 days to 4 years). There were two male and four female children in our study.

Of six patients, one presented with Abernethy malformation type la, two with type $1 b$, and three with type 2. Four of the six patients presented with CEPS-related complications; whereas, two patients were asymptomatic. One patient presented with cholestatic jaundice and large hepatic mass at 2 months after birth. The investigations for other causes of cholestasis showed negative results. The abdominal US revealed a single shunt and an intrahepatic mass, which manifested as focal nodular hyperplasia on biopsy. Three elder children, aging from

Table 1. Classification of Congenital Extrahepatic Portosystemic Shunt

\begin{tabular}{cl}
\hline Classification & Definition \\
\hline Type 1 & Absence of intrahepatic portal veins \\
Type la & Superior mesenteric and splenic vein drain separately into inferior vena cava \\
Type lb & Superior mesenteric vein and splenic vein form a common trunk before draining into the inferior vena cava \\
Type 2 & Important collateral, patent intrahepatic veins \\
\hline
\end{tabular}




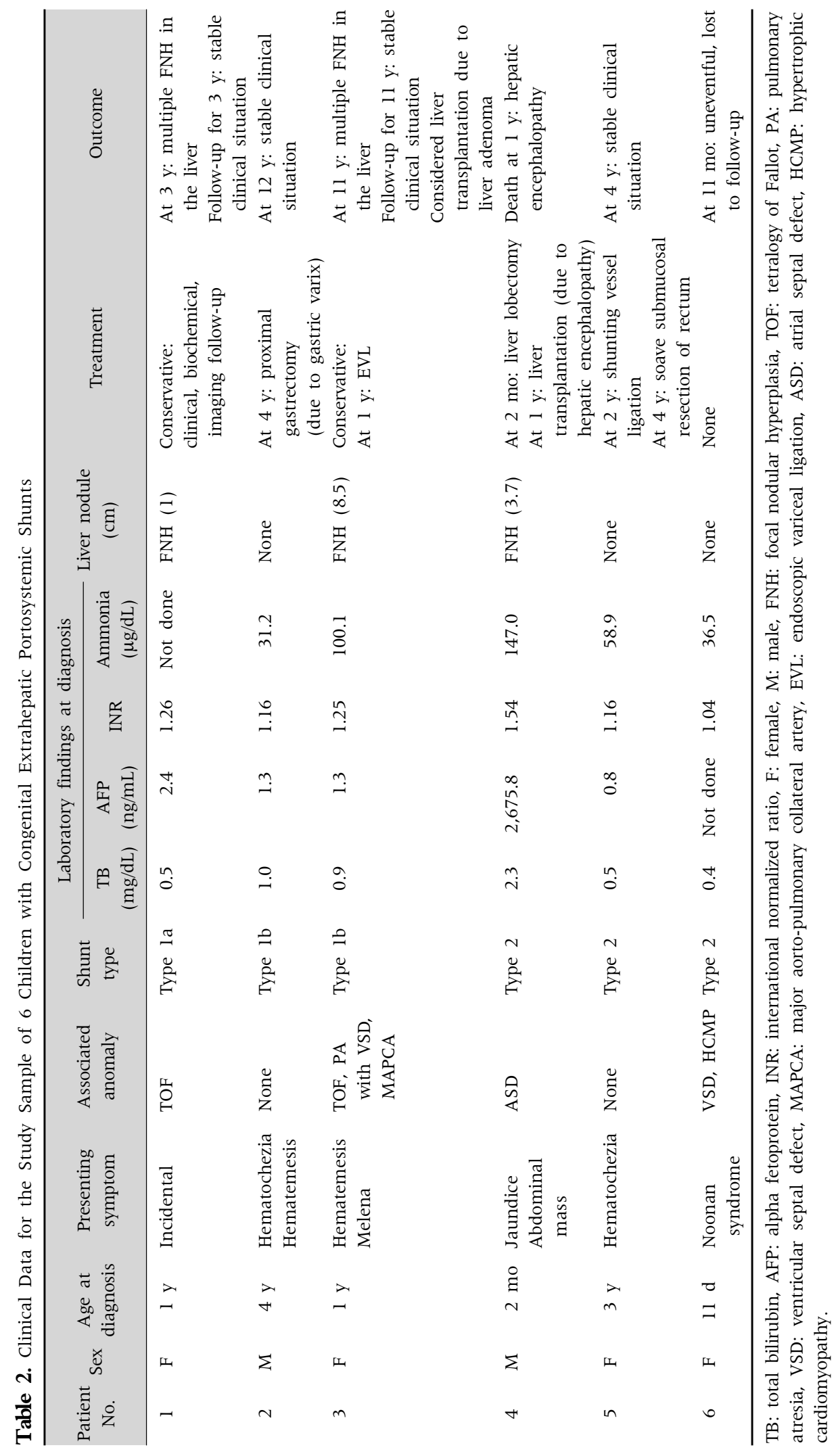


1 to 4 years, presented with an initial symptom of gastrointestinal bleeding (hematemesis, melena, hematochezia). In these patients, the imaging studies with abdominal CT revealed esophageal varices or varicose veins in the perirectal region. In the remaining 2 children who presented with urinary tract infection and congenital hypertrophic cardiomyopathy associated with Noonan syndrome, the portosystemic shunts were found in abdominal US incidentally.

Liver function tests showed elevated serum aspartate aminotransferase and alanine aminotransferase in 3 patients, abnormal prothrombin time in 5 patients, and elevated blood ammonia in 3 patients. At presentation, elevated alpha-fetoprotein levels and liver mass were reported in only one patient, who underwent right lobectomy for focal nodular hyperplasia. In addition, 3 children presented with benign liver mass, which were suspected as focal nodular hyperplasia, with diameter ranging from 1.0 to $8.5 \mathrm{~cm}$.

Among three patients with type l CEPS, two patients are waiting for liver transplantation because of increasing the number of liver tumors and one patient is in stable clinical situation with regular screening tests for associated morbidities.

In patients with type 2 CEPS, one patient was planned on surgical shunting vessel ligation, but the patient suddenly developed hepatic encephalopathy, and died of acute hepatic failure, although he underwent a liver transplantation [7]. Another patient diagnosed with CEPS type 2 underwent shunting vessel ligation allowing for age, 2 years-old, and anatomy of the shunt. While the third patient had been in stable clinical situation but was lost to follow-up.

\section{DISCUSSION}

In embryonic period, epithelial and mesenchymal interactions occur in the network of early embryonic and then fetal vessels, which involute both inside and outside the liver. Abnormal vascular communications can occur between any vein of the portal system and any branch of the inferior vena cava because of the incomplete involution of one or several of these primordial vessels $[8,9]$. These abnormal struc- tures, like extrahepatic portal veins and ductus venosus, can persist throughout life and give rise to risks of complications. Severe complications are neonatal cholestasis; hyperammonemia and encephalopathy (35\%); benign and malignant liver tumors (26\%); hepatopulmonary syndrome and porto-pulmonary hypertension (18\%) [10,11].

Since the initial description about Abernethy malformation, formerly called CEPS, only about 80 cases have been reported, with most of the patients under the age of 18 years [12]. As reported earlier, type 1 malformations are predominantly found in females $(74 \%)$, and are more associated with other congenital defects than type 2 CEPS, such as congenital heart disease, genitourinary disease, skeletal abnormalities, etc. Our study reported more number of female patients $(66.7 \%)$ who presented with type 1 CEPS, and all those patients also presented with other associated congenital defects (Table 2 ).

We compared our cases with other such cases reported in France and China (Table 3 ) $[5,7,8]$. In our

Table 3. Comparison of the Patients of CEPS in Korea, China, France

\begin{tabular}{lccc}
\hline & Korea & China & France \\
\hline $\begin{array}{l}\text { No. of patient } \\
\text { Shunt type }\end{array}$ & 6 & 7 & 11 \\
$\quad$ Type 1 & 50.0 & 42.9 & 36.4 \\
$\quad$ Type 2 & 50.0 & 57.1 & 63.6 \\
Symptoms & & & \\
$\quad$ Gastrointestinal bleeding & 50.0 & 85.7 & 0 \\
$\quad$ Jaundice & $16.7^{\dagger}$ & 0 & 18.2 \\
$\quad$ Abdominal mass & $16.7^{\dagger}$ & 0 & 18.2 \\
CNS symptoms* & $16.7^{\dagger}$ & 0 & 27.3 \\
$\quad$ Dyspnea & 0 & 0 & 27.3 \\
$\quad$ Incidental & 33.3 & 14.3 & 9.1 \\
Liver nodules & 50.0 & 71.4 & 18.2 \\
Treatment & & & \\
$\quad$ Liver transplantation & 16.7 & 0 & 9.1 \\
$\quad$ Shunt ligation/closure & 16.7 & 0 & 54.5 \\
$\quad$ Conservative & 66.6 & 100 & 36.4 \\
Outcome, death & 16.7 & Unknown & 9.1 \\
\hline
\end{tabular}

Values are presented as number of patients or percent data. CNS: central nervous system.

*Mental retardation, drowsiness during day, portal systemic encephalopathy.

${ }^{\dagger}$ Jaundice, abdominal mass and hepatic encephalopathy were presented in one patient simultaneously. 
study, there was no remarkable difference in the incidence of type 1 and 2 CEPS; on the other hand, in China and France, the incidence of type 2 malformation was higher. At presentation, the most common initial symptom was gastrointestinal bleeding followed by central nervous system (CNS) symptoms, which included mental retardation, drowsiness, and portal systemic encephalopathy. Abdominal mass, which was confirmed by imaging studies, jaundice, and dyspnea were also frequently found as initial presenting symptoms. Since numerous developing collaterals, such as the superior hemorrhoidal plexus and varicose veins in the perirectal region may cause hemorrhoidal bleeding, gastrointestinal bleeding is speculated as the most common symptom of CEPS [3]. In China, the most common presenting symptom was also gastrointestinal bleeding, but in France, the most frequent complications were dyspnea and CNS symptoms.

In our study, one patient (16.7\%) with type 2 CEPS was treated by liver transplantation, and another patient $(16.7 \%)$ with type 2 CEPS was treated by shunting vessel ligation, while rest of the patients were regularly followed-up with conservative treatment. In China, all patients were managed by conservative treatment; while in France, 6 patients (54.5\%) were treated by shunt ligation or closure, 1 patient $(9.1 \%)$ was treated by liver transplantation, and 4 patients $(36.4 \%)$ were treated by conservative treatment.

The most notable differentiating point is that, the proportion of active treatment varies from country to country. One of three patients with type 2 CEPS in Korea $(33.3 \%)$, six of seven patients with type 2 CEPS $(85.7 \%)$ in France, none in China $(0 \%)$, were treated by shunt ligation or closure. However, since long-term and large-sample studies have not yet been published, we do not know the difference of life-long outcome among the methods of treating CEPS.

Many other studies have reported that, early recognition is important because liver nodules may develop into hepatic neoplasms [13], and hepatic encephalopathy may occur suddenly [8], as in one of type 2 CEPS patients in our case. There are no guidelines yet for advising earlier treatment of CEPS to prevent the development of complications [6]. However, a few literatures about Abernethy malformation emphasize that when CEPS is suspected, the diagnosis should be confirmed immediately, and complications should be investigated to start early treatment for restoring portal flow and preventing complications [14]. The treatment options for CEPS depend on the type of shunts, the anatomic situation of the intrahepatic portal vein, condition of the patient, and local expertise $[15,16]$. In type 1 CEPS, the only effective treatment option is liver transplantation, because occlusion of the shunt will obstruct the only drainage route of the mesenteric venous blood $[8,17]$; however, transplantation should be performed only in cases with exceptionally complex anatomy where closure of the shunt is not possible. Patients with type 2 CEPS with serious symptoms such as hepatic encephalopathy, may benefit from early shunt closure, in one or two steps, depending on the level of portal pressure assessed by an occlusion test $[13,16,18,19]$. Owing to the severity of complications and their potential reversibility after suppression of the shunts, closure or ligation of a shunt in type 2 CEPS is necessary [7]. It is performed either surgically or with percutaneous transcatheter embolization [20]. Various studies have strongly recommended that, when a shunt is detected with no clinically significant complications and is confirmed to be permanent, it should be closed before a significant complication occurs if a closure can be performed satisfactorily $[8,21]$.

Since CEPS is a rare disease, and only small-sample studies have been reported, the disease often remains underestimated. In patients with unexplained signs and symptoms of gastrointestinal bleeding, encephalopathy, liver mass, porto-pulmonary hypertension, or hepatopulmonary syndrome, and especially in cases with chromosomal syndromes such as Noonan's syndrome, the associated presence of CEPS needs further consideration. Finally, clinical diagnosis must be carried out by radiologic evaluation, which is of primary importance to confirm the diagnosis and to plan management, thus avoiding the most severe consequences of this malformation. 
The present study has a few limitations. Our study was limited to a relatively small number of patients in a single center, and therefore, the study population may not be representative of all pediatric patients in Korea. Furthermore, comparative groups of China and France were also small sized cohorts, hence these studies may also not be representative of all pediatric patients in each of these countries. Large-sample studies including CEPS are needed to evaluate the characteristics of patients with CEPS furthermore and determine the treatment guidelines for CEPS.

\section{REFERENCES}

1. Kumar A, Kumar J, Aggarwal R, Srivastava S. Abernethy malformation with portal vein aneurysm. Diagn Interv Radiol 2008;14:143-6.

2. Burdall OC, Grammatikopoulos T, Sellars M, Hadzic N, Davenport M. Congenital vascular malformations of the liver: an association with trisomy 21. J Pediatr Gastroenterol Nutr 2016;63:e141-6.

3. Niwa T, Aida N, Tachibana K, Shinkai M, Ohhama Y, Fujita K, et al. Congenital absence of the portal vein: clinical and radiologic findings. J Comput Assist Tomogr 2002;26:681-6.

4. Yi JE, Jung HO, Youn HJ, Choi JY, Chun HJ, Lee JY. A case of pulmonary arterial hypertension associated with congenital extrahepatic portocaval shunt. J Korean Med Sci 2014;29:604-8.

5. Gong Y, Zhu H, Chen J, Chen Q, Ji M, Pa M, et al. Congenital portosystemic shunts with and without gastrointestinal bleeding - case series. Pediatr Radiol 2015; 45:1964-71.

6. Timpanaro T, Passanisi S, Sauna A, Trombatore C, Pennisi M, Petrillo G, et al. Congenital portosystemic shunt: our experience. Case Rep Pediatr 2015;2015: 691618.

7. Lee HJ, Lee JH, Huh J, Kang IS, Lee HJ, Suh YL, et al. A case of congenital extra hepatic portocaval shunt(abernethy malformation type 2) with a very largeliver mass and an atrial septal defect. Korean J Pediatr Gastroenterol Nutr 2008;11:56-9.

8. Franchi-Abella S, Branchereau S, Lambert V, Fabre M, Steimberg C, Losay J, et al. Complications of congenital portosystemic shunts in children: therapeutic options and outcomes. J Pediatr Gastroenterol Nutr 2010;51: 322-30.
9. Scalabre A, Gorincour G, Hery G, Gamerre M, Guys JM, de Lagausie P. Evolution of congenital malformations of the umbilical-portal-hepatic venous system. J Pediatr Surg 2012;47:1490-5.

10. Yazal Erdem A, Emir S, Demir HA, Özyörük D, Çetin İ, Turan A, et al. Focal nodulary hyperplasia of the liver due to congenital portosystemic shunt: a rare condition mimicking hepatocellulary carcinoma. J Pediatr Hematol Oncol 2017;39:e8-10.

11. Kim MJ, Ko JS, Seo JK, Yang HR, Chang JY, Kim GB, et al. Clinical features of congenital portosystemic shunt in children. Eur J Pediatr 2012;171:395-400.

12. Mistinova J, Valacsai F, Varga I. Congenital absence of the portal vein--Case report and a review of literature. Clin Anat 2010;23:750-8.

13. Sharma R, Suddle A, Quaglia A, Peddu P, Karani J, Satyadas T, et al. Congenital extrahepatic portosystemic shunt complicated by the development of hepatocellular carcinoma. Hepatobiliary Pancreat Dis Int 2015;14:552-7.

14. Kwapisz L, Wells MM, AlJudaibi B. Abernethy malformation: congenital absence of the portal vein. Can J Gastroenterol Hepatol 2014;28:587-8.

15. Kong Y, Zhang H, Liu C, Wu D, He X, Xiao M, et al. Abernethy malformation with multiple aneurysms: incidentally found in an adult woman with Caroli's disease. Ann Hepatol 2013;12:327-31.

16. Fu L, Wang Q, Wu J, Guo Y, Huang M, Liu T, et al. Congenital extrahepatic portosystemic shunt: an underdiagnosed but treatable cause of hepatopulmonary syndrome. Eur J Pediatr 2016;175:195-201.

17. Charre L, Roggen F, Lemaire J, Mathijs J, Goffette P, Danse E, et al. Hematochezia and congenital extrahepatic portocaval shunt with absent portal vein: successful treatment by liver transplantation. Transplantation 2004;78:1404-6.

18. Pathak A, Agarwal N, Mandliya J, Gehlot P, Dhaneria M. Abernethy malformation: a case report. BMC Pediatr 2012;12:57.

19. Bernard O, Franchi-Abella S, Branchereau S, Pariente D, Gauthier F, Jacquemin E. Congenital portosystemic shunts in children: recognition, evaluation, and management. Semin Liver Dis 2012;32:273-87.

20. Zhang B, Wu KT, Li L, Lai HY. Catheter embolization of type II congenital extrahepatic portosystemic shunt with hematochezia: a case series and review of the literature. Cardiovasc Intervent Radiol 2018;41:1121-7.

21. Sanada Y, Urahashi T, Ihara Y, Wakiya T, Okada N, Yamada N, et al. The role of operative intervention in management of congenital extrahepatic portosystemic shunt. Surgery 2012;151:404-11. 